\title{
Remote Photoplethysmography for Evaluation of Cutaneous Sensory Nerve Fiber Function ${ }^{\dagger}$
}

\author{
Zbignevs Marcinkevics ${ }^{1,2, *}$, Alise Aglinska ${ }^{2}$, Uldis Rubins ${ }^{2}$ and Andris Grabovskis ${ }^{2}$ \\ 1 Department of Human and Animal Physiology, Faculty of Biology, University of Latvia, Jelgavas St.1, \\ LV-1004 Riga, Latvia \\ 2 Biophotonics Laboratory, Institute of Atomic Physics and Spectroscopy, University of Latvia, Jelgavas St. 3, \\ LV-1004 Riga, Latvia; aglinska96@gmail.com (A.A.); uldis.rubins@lu.lv (U.R.); \\ andris.grabovskis@gmail.com (A.G.) \\ * Correspondence: zbigis@latnet.lv \\ $+\quad$ This paper is an extended version of our paper published in Marcinkevics, Z.; Aglinska, A.; Rubins, U.; \\ Mikale, A.; Grabovskis, A. Imaging photoplethysmography for evaluation of cutaneous sensory nerve fiber \\ function. In Proceedings of the 3rd International Conference "Biophotonics-Riga" 2020, LV-1004, Riga, Latvia, \\ 24-25 August 2020.
}

check for

updates

Citation: Marcinkevics, Z.; Aglinska,

A.; Rubins, U.; Grabovskis, A. Remote

Photoplethysmography for

Evaluation of Cutaneous Sensory

Nerve Fiber Function. Sensors 2021,

21, 1272. https://doi.org/

doi:10.3390/s21041272

Academic Editor: Sabina Merlo

Received: 4 January 2021

Accepted: 8 February 2021

Published: 11 February 2021

Publisher's Note: MDPI stays neutral with regard to jurisdictional claims in published maps and institutional affiliations.

Copyright: (c) 2021 by the authors. Licensee MDPI, Basel, Switzerland. This article is an open access article distributed under the terms and conditions of the Creative Commons Attribution (CC BY) license (https:/ / creativecommons.org/licenses/by/ $4.0 /)$.
Abstract: About $2 \%$ of the world's population suffers from small nerve fiber dysfunction, neuropathy, which can result in severe pain. This condition is caused by damage to the small nerve fibers and its assessment is challenging, due to the lack of simple and objective diagnostic techniques. The present study aimed to develop a contactless photoplethysmography system using simple instrumentation, for objective and non-invasive assessment of small cutaneous sensory nerve fiber function. The approach is based on the use of contactless photoplethysmography for the characterization of skin flowmotions and topical heating evoked vasomotor responses. The feasibility of the technique was evaluated on volunteers $(n=14)$ using skin topical anesthesia, which is able to produce temporary alterations of cutaneous nerve fibers function. In the treated skin region in comparison to intact skin: neurogenic and endothelial component of flowmotions decreased by $\sim 61 \%$ and $41 \%$, the local heating evoked flare area decreased by $\sim 44 \%$, vasomotor response trend peak and nadir were substantially reduced. The results indicate for the potential of the remote photoplethysmography in the assessment of the cutaneous nerve fiber function. It is believed that in the future this technique could be used in the clinics as an affordable alternative to laser Doppler imaging technique.

Keywords: cutaneous perfusion; remote photoplethysmography; chronic pain; neuropathic pain; pain assessment; cutaneous flowmotions; vasomotor response; topical heating

\section{Introduction}

Small fiber peripheral neuropathy is a chronic nerve disorder, which occurs when damage to the peripheral nerves predominantly or entirely affects the small myelinated (A $\delta$ ) fibers or unmyelinated C fibers, which are involved in various somatic and autonomic functions [1], but mainly the sensory functions account for its manifestation such as thermal perception and nociception [2]. Therefore, the most debilitating symptoms of these impairments are ongoing burning pain, stabbing pain, squeezing or pressure pain, paroxysmal electric shock-like sensations, or mechanical dynamic allodynia [3], leading to a marked deterioration of the patients' quality of life.

The exact prevalence of painful peripheral small fibers neuropathy is not known, as the disorder is associated with many diseases, including diabetes, infections, autoimmune and endocrine disorders, but it can also occur due to genetic alterations. In a review from 2015, Callaghan et al. reported that about $2-7 \%$ of the worldwide population might be affected by neuropathy [4] and this number in the near future may increase due to Coronavirus disease (COVID-19) neurological complications which affect both the central and peripheral nervous system [5-7]. 
Currently, the most common way to confirm small sensory nerve fiber damage is the quantitative somatosensory testing (QST) [8], which is a subjective and time-consuming method, or skin biopsy $[9,10]$, which is invasive and uncomfortable for the patients. Among potential body sites suitable for diagnostics of chronic disease, the most promising is human skin, which is the largest organ, strategically located at the interface with the external environment with its complex diffuse neuro-immuno-endocrine system [11], which is able to represent the situation in the whole body [12,13], potentially serving as an early diagnostic marker [13]. In terms of the diagnostic side, the favorable feature of the skin is its immediate and complete accessibility for optic diagnostic techniques, partly due to superficial location, partly favorable optical properties, such as relatively large light penetration depth [14]. However, the challenging part is the fact that the structure of the skin is rather complicated [15], optical properties are not uniform [16] and may alter depending on the condition of regulatory mechanisms which are not fully elucidated. Thus, non-invasive assessment of cutaneous peripheral nerve fiber function is still challenging due to the current lack of simple and objective diagnostic techniques. Therefore, the development of objective methods is essential for diagnostics and disease course monitoring.

There are studies confirming the application of cutaneous microvascular bed for small nerve fiber function assessment, both during resting conditions using spectral analysis of blood perfusion oscillations (flowmotions) [17] and by examination of cutaneous perfusion in response to various provocation tests (reactivity tests) [18-24] which involve topical skin heating, topical skin cooling, application of mechanical compression and electrical stimuli [17]. This approach is based on the ability of cutaneous nerve fibers to mediate local vascular tone, which is manifested as blood perfusion changes. Despite various studies confirming the potential of skin for assessment of peripheral nervous system functions, such tests are still not used by clinicians as a routine procedure, which is partly due to the lack of reliability, specificity and sensitivity, partly due to the absence of simple and cost-effective instrumentation, the latter is supported by the fact that cutaneous perfusion has been extensively examined using Laser Doppler flowmetry technique, which is relatively expensive and therefore limited to a wide range of clinicians [25] including general practitioners. The cost-effective alternative could be remote photoplethysmography [26], which is a contactless optical technique for blood volume pulsation detection in the tissue using various computational algorithms [27] and relatively simple instrumentation: video camera, to detect subtle variations of back-reflected light, and appropriate light source. Currently, the imaging photoplethysmography (iPPG) is expanding its applications beyond the assessment of heart rate and arterial stiffness, illustrated by previous studies such as studies of Kamshilin et al. suggesting the high clinical potential of remote photoplethysmography in the evaluation of cutaneous vasomotor responses [27-32]. However, further refinement of this approach requires alteration of small sensory nerve fiber function in a controllable manner, which is difficult to achieve in neuropathic patients due to differences in etiology, degree of dysfunction, comorbidities, and other factors [33]. Therefore, the development of novel protocols mimicking peripheral nervous system dysfunction in healthy subjects for the assessment of optical techniques is of great importance.

The present study aimed to evaluate the feasibility of remote photoplethysmography for the assessment of small cutaneous sensory nerve fiber function on healthy young volunteers, using novel skin topical anesthesia protocol. It has been hypothesized that remote plethysmography can provide information related to cutaneous nerve fiber function.

\section{Materials and Methods}

\subsection{Remote Photoplethysmography Setup}

The major components of the developed photoplethysmography system were as follows: monochromatic camera USB-3.0, ADC 8-12-bits, resolution $648 \times 488$ pix. (Ximea MC023MG-SY-UB, XIMEA GmbH., Münster, Germany) with $540 \mathrm{~nm}-10 \mathrm{~nm}$ bandpass narrowband interference filter (Edmund Optics GmbH., Mainz, Germany), white industrial 
LED light source 50W (V-TAC, Shenzhen, China) and orthogonal polarizers film to reduce surface reflection. The choice of narrowband interference filter was based on our prior study, [30] confirming substantial improvement of signal quality as $540 \mathrm{~nm}$ corresponds to one of the Oxyhemoglobin absorption peaks.

Skin topical heating was performed using medical grade heater-thermostat (Moor VMS-HEAT, Moore Instruments, Ltd., Devon, UK) equipped with two heating probes (VHP1 and VHP3, Moore Instruments, Ltd., Devon, UK), and video data stored on the laptop computer (Dell Latitude 5510, Dell. Inc., Austin, TX, USA), see Figure 1. The video signal was acquired using a laptop computer operating Ximea software and stored to an uncompressed avi file.

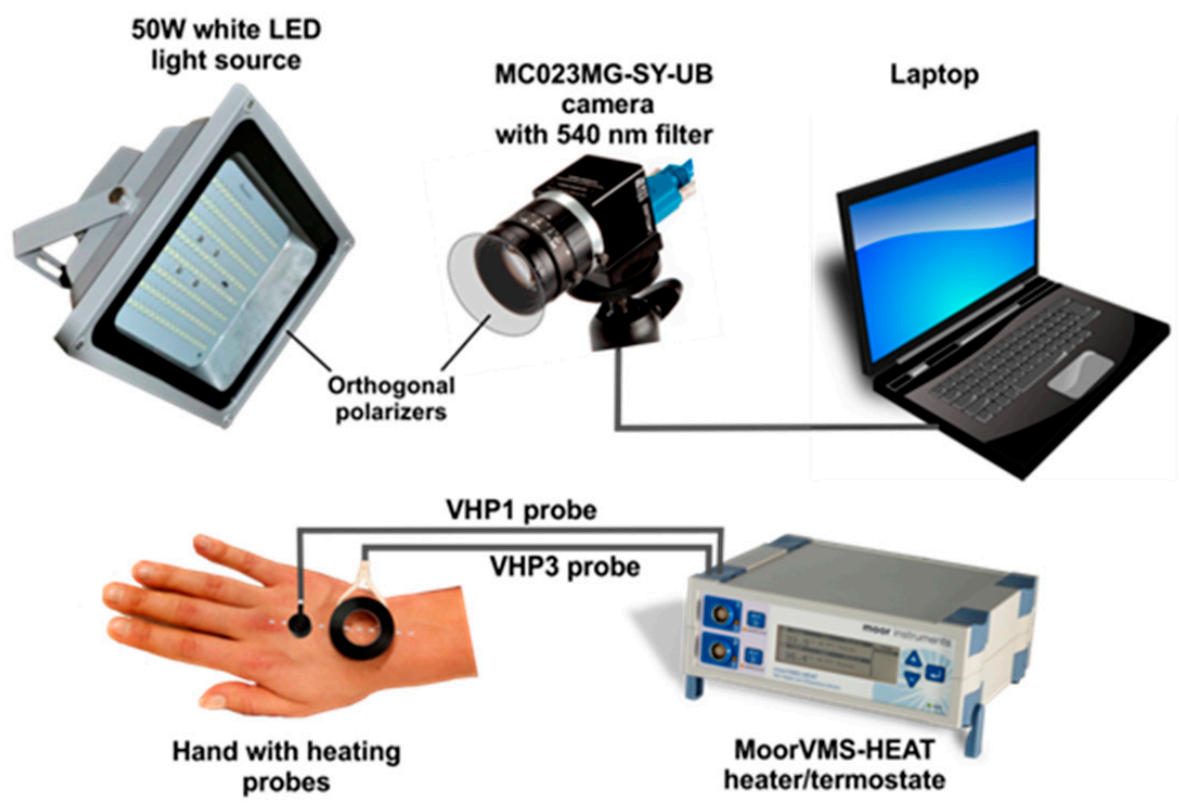

Figure 1. Remote photoplethysmography setup and used equipment; Palm dorsal aspect middle line is denoted by white dotted line.

\subsection{Measurement Protocol}

Fourteen (12 females and 2 males) clinically healthy volunteers (20-40 y) were recruited from the University of Latvia community. The study procedures were approved by the Ethics Committee of the University of Latvia, Institute of Cardiology and Regenerative Medicine (Prot.Nr: 03.05.2018), and was in accordance to the Declaration of Helsinki [34]. Prior to the study, all subjects were informed about the protocol and gave written informed consent. All measurements were performed at $21 \pm 1^{\circ} \mathrm{C}$ in a dark, well-ventilated room. The whole procedure comprised two consecutive protocols: Induction of temporal cutaneous nerve fiber dysfunction and skin topical heating. To simulate neuropathic alterations of small cutaneous sensory nerve fibers, skin topical anesthesia protocol described in Green et al., 2009 [24] was modified, so that control and anesthetized regions were situated close to each other on the same hand, with the advantage to acquire data from both sites simultaneously in one single frame using the same imaging system. The detailed description of our protocol is described below.

One hour before topical heating, the dorsal surface of the subject's hand was divided into two regions by an imaginary middle line (Figure 1), on the left was the control regionwith intact skin, and on the right was the region where an anesthetic substance (EMLA 5\% Cream, AstraZeneca, Södertälje, Sweden) was carefully applied, so-called anesthetized region, Figure 2a. Following an hour exposure time, anesthetic cream was gently removed with 70\% isopropyl alcohol whips (Alcohol Preps, Romed Inc., Wilnis, The Netherlands) skin was allowed to dry and the subject was asked to lie on the surgical chair (Mars 2, TRUMPF Medizin Systeme GmbH, Saalfeld, Germany) comfortably in a supine position 
with hand supported by vacuum pillow (AB Germa, Kristianstad, Sweden). To produce a topical heating induced response, the central point of VHP1 and VHP3 heating probes were placed on the imaginary line (midline), so that the same probe contacted anesthetized and intact skin regions. The VHP3 probe was attached to the skin using self-adhesive ring shaped tape and filled with distillated water, whereas VHP1 probe was gently placed on the skin and secured with the thin rubber belt as seen in Figure 1. Baseline perfusion was achieved by heating skin at 32 degrees for $1 \mathrm{~min}$, while topical heating induced response was produced by heating skin at 42 degrees for $19 \mathrm{~min}$. During the protocol, the video of the entire dorsal aspect of the hand was captured with monochromatic camera using manual exposure at $25 \mathrm{fps}$ for $23 \mathrm{~min}$, which later allowed selecting several separate regions of interest for offline analyses.

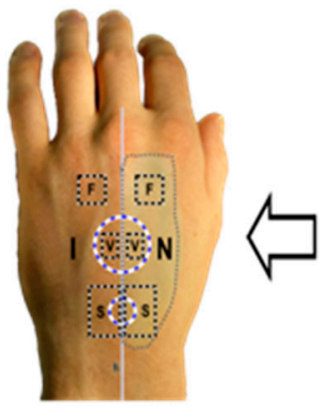

(a)

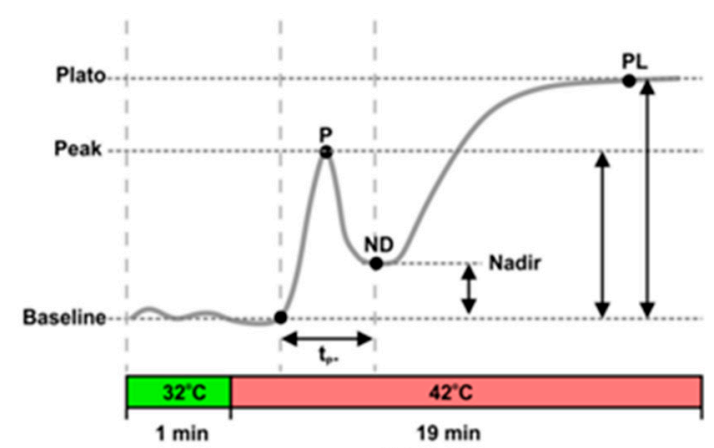

(b)

Figure 2. (a)-Position of probes and selected regions of interest following application of EMLA cream; I-intact skin region, N-EMLA gel numbed skin region. Position of the VHP3 and VHP1 probes are marked by large and small, blue dotted circles. Six regions of interest are indicated by black dotted squares: Flowmotion detection (F), vasomotor response trend (V) and flare area (S). (b)-Timeline of topical skin heating and typical trend of evoked vasomotor response; P-first peak, ND-nadir, PL-plato phase, tp-peak duration.

\subsection{Signal Processing}

Recorded videos were processed offline using custom designed MATLAB (Mathworks Inc., Novi, MI, USA) software (rPPG analyses, author: U. Rubins, University of Latvia, Riga, Latvia). The software performs computation of rPPG signals from recorded video file and calculates fast-varying (AC) and slow-varying (DC) rPPG components in order to detect dynamics of hemodynamic parameters, such as heart rate and blood volume changes in cutaneous tissue.

To compute skin repetitive low frequency (LF) oscillations, or so-called microvascular flowmotion which represent the influence of myogenic $(0.05-0.15 \mathrm{~Hz})$ [35], neurogenic $(0.02-$ $0.05 \mathrm{~Hz})[36]$ and endothelial $(0.0095-0.02 \mathrm{~Hz})[37,38]$ activity on vascular tone, temporal component was filtered using 2nd order Butterworth zero-phase digital band-pass filter. To extract heartbeat-related fast-varying rPPG AC perfusion component, the signal was filtered in the same way in a frequency range $0.6-6 \mathrm{~Hz}$.

During the analysis, the RoI is manually chosen over the skin region where the manipulations were done. The software calculates spatially averaged rPPG signal. Spatially averaged filtered rPPG signal can be found as:

$$
R_{n}=\frac{1}{M} \sum_{j=1}^{M} S_{j, n}
$$

where $R n$ is spatially averaged signal $S_{j, n}$ obtained in $j$-th pixel in $n$-th video frame, in RoI containing $M$ pixels. To obtain the set of perfusion parameters, the single-period waveform 
is calculated by beat-per-beat manner. The feet of waveforms are detected, by finding local minima. Then the set of perfusion index $(P I)$ values is calculated in the following way:

$$
P I_{k}=100 R_{k} / D C_{0, k}
$$

where $P I_{k}$ is perfusion index, $D C_{0, k}$ is zero-frequency DC signal, which are calculated in $k$-th beat of rPPG waveform. PI( $t)$ signal was calculated in every heartbeat. To remove noise and outliers from PI signal, built in Matlab Hampel filter was used, functioning in the following way: for each sample of $P I$, the function computes the median of a window composed of the sample and its $\mathrm{N}$ surrounding samples. The standard deviation of each sample is estimated about its window median using the median absolute deviation. If a sample differs from the median by more than $\mathrm{S}$ standard deviations, it is replaced with the median value. For our measurements, we chose $\mathrm{N}=30$ and $\mathrm{S}=0.1$.

To obtain spatial distribution of blood perfusion in skin tissue perfusion map (AC or DC) was calculated using the $R_{n}$ as the ground truth reference signal by Pearson's linear correlation coefficient matrix. The correlation was computed between the rPPG signal obtained in each pixel of image and the reference signal to determine the signal strength in each pixel of video:

$$
\operatorname{map}_{j}=\frac{\sum_{n=1}^{N}\left(S_{j, n}-\overline{S_{j, n}}\right)\left(R_{n}-\overline{R_{n}}\right)}{\sqrt{\sum_{n=1}^{N}\left(S_{j, n}-\overline{S_{j, n}}\right)^{2}} \sqrt{\sum_{n=1}^{N}\left(R_{n}-\overline{R_{n}}\right)^{2}}}
$$

where $n$ is a frame count in video buffer, $S_{j, n}$ is a rPPG signal obtained in $j$-th pixel of $n$-th video frame, $R_{n}$ is the reference signal.

To compute cutaneous repetitive low frequency (LF) oscillations, the power spectrum density of band-pass filtered rPPG signal was calculated for three aforementioned spectral intervals using 16 min recording in $40 \times 40$ pixel RoI window. The power density was calculated in a following way:

$$
P D=\frac{1}{N} \sum_{n=1}^{N} \operatorname{Re}[F\{R\}]_{n}
$$

where $P D$ is a power density, $\operatorname{Re}[F\{R\}]$ is a real part of Fourier transformed RoI-averaged signal, $N$-number of samples.

\subsection{Analyses of Cutaneous Perfusion Data}

The acquisition of the video from the entire dorsal aspect of the palm allowed selecting six regions of interest for further processing of cutaneous perfusion data. Characterization of vasomotor response trend was performed by selecting two regions inside VHP3 heating probe transparent part- one from intact skin, and one from anesthetized skin, and perfusion index changes over the time computed, Figure 2a. Four parameters were calculated from denoized perfusion index datasets: First peak amplitude (P), amplitude of nadir (ND), plato phase amplitude (PL) and first peak duration (tp) as depicted in Figure $2 \mathrm{~b}$.

To explore flare response, two regions of interest surrounding VHP1 probe were selected (Figure 2a) and analyses performed on one-minute duration video fragment just following removal of the probe. Offline analyses included the generation of perfusion map, with the following determination of flare area in intact and anesthetized regions.

Cutaneous flowmotions were calculated from flare and heating unaffected skin, by selecting two $\sim 40 \times 40$ pixel regions one from intact and the other from anesthetized skin in 16 min duration video fragment as depicted in Figure 2a. Flowmotions were divided into the three spectral ranges representing the influence of myogenic $(\sim 0.05-0.15 \mathrm{~Hz})$ [35], neurogenic $(\sim 0.02-0.05 \mathrm{~Hz})[36]$ and endothelial $(\sim 0.0095-0.02 \mathrm{~Hz})[37,38]$ activity as suggested by other studies, and spectral power for each range was computed using Fast Fourier Transformas was described in the signal processing section. 


\subsection{Statistical Analyses}

Statistical analyses were performed using SigmaPlot 12.0 (Systat Software Inc., San Jose, CA, USA). The data followed Gaussian distribution (Shapiro-Wilk test) therefore parametric statistical tests have been considered. The data of topical skin heating vasomotor response, and cutaneous flowmotions were analyzed using paired T-test and One-WayRepeated Measure Analyses of Variance (One Way RM ANOVA), while the comparison of flare area in the control and EMLA treated regions were performed by paired T-test. Statistically significant difference is considered at $p<0.05$. The values in the graphs are expressed as arithmetic mean \pm standard error unless stated otherwise.

\section{Results}

\subsection{Topical Heating Induced Flare Response}

After removal of the VHP1 probe, the typical reddening of skin surrounding the probe (flare) was observed in all subjects, in both intact and anesthetized skin regions.

Nevertheless, the significantly large flare area was observed in intact skin as shown in Figure 3a. The group mean flare area in intact skin region was $114.59 \pm 59.03 \mathrm{~mm}^{2}$, and in the anesthetized region (EMLA treated region) was $41.30 \pm 26.50 \mathrm{~mm}^{2}$ pointing out a substantial difference between intact and EMLA affected skin, with an average difference $73.30 \pm 47.50 \mathrm{~mm}^{2}$ which correspond to $59.60 \pm 17.90$ percent changes, see Figure $3 \mathrm{~b}$.
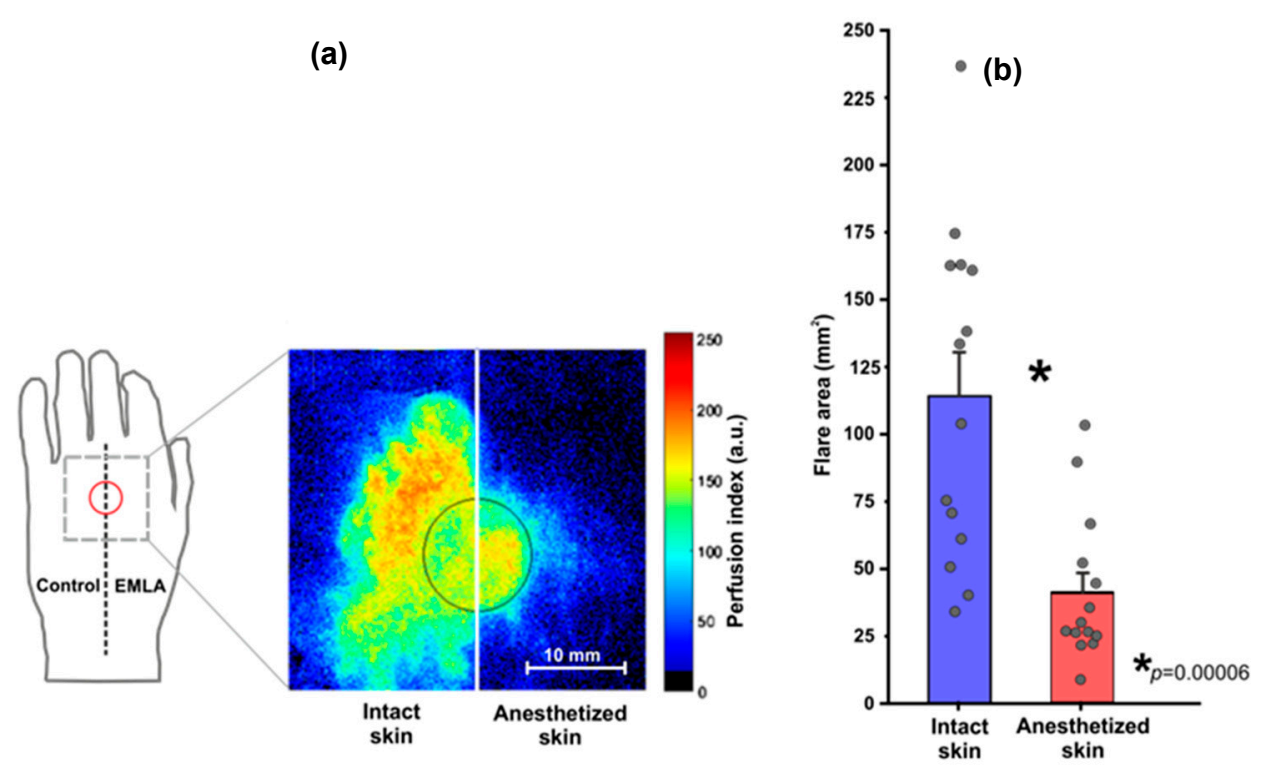

Figure 3. (a)-Perfusion index map exhibiting topical heating evoked flare response in intact and EMLA treated skin sitecircle indicates position of VHP1 probe on the dorsal aspect of palm; typical example from one subject. (b)-Comparison of flare area in intact and EMLA treated skin region, group $(n=14)$ mean \pm S.E.M., subjects individual measurements marked with black small circles. Statistically significant difference is denoted by asterisk.

\subsection{Topical Heating Induced Vasomotor Response Trend}

The typical vasomotor response, reported in other studies using laser Doppler flowmetry, was observed in the intact skin region during the skin topical heating test, comprising baseline perfusion during preheating, rapid increase of perfusion, the so-called first peak with the following nadir and relatively steady plato phase as depicted in Figure 4a. 

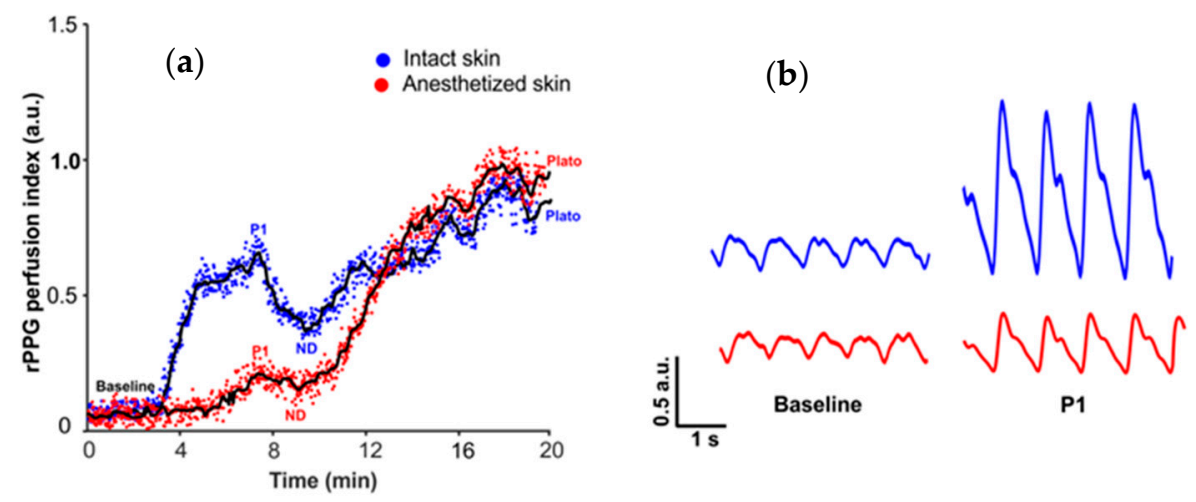

Figure 4. (a)-Topical skin heating (VHP3 probe) induced vasomotor response in intact and anesthetized skin; representative example from one subject, Characteristic parameters of vasomotor response are depicted on the graph: P1-first peak, ND-nadir. (b)-Example of photoplethysmography (rPPG) waveform during baseline and peak (P) from intact and EMLA treated skin (anesthetized skin); Example from one subject.

While in the EMLA treated skin region, different vasomotor response was observed: The duration and amplitude of initial peak and nadir was substantially diminished, hence parameters of baseline perfusion and plato phase remained unchanged, (Figure 4a)-which is a noteworthy observation supporting the contribution of neurogenic mechanisms in formation of the initial peak.

In the intact skin region the baseline perfusion was $0.27 \pm 0.21$ a.u., the initial peak amplitude reached $1.35 \pm 0.36$ a.u., while in EMLA treated region baseline perfusion was $0.21 \pm 0.12$ a.u. and initial peak amplitude was $0.68 \pm 0.27$ a.u. Similarly, in the intact region nadir amplitude was $0.71 \pm 0.27$ a.u. but in anesthetized region $0.41 \pm 0.23$ a.u. as depicted in Figure 5a. Another observed effect of EMLA cream was an increase of initial peak duration: in intact skin duration was $6.99 \pm 1.90 \mathrm{~min}$, but in anesthetized region $4.99 \pm 2.08 \mathrm{~min}$, as shown in Figure $5 \mathrm{~b}$. The observed effects of EMLA gel on cutaneous blood perfusion during topical heating is mostly attributed to diminished amplitude of photoplethysmography AC component as depicted in Figure $4 b$, which largely determines perfusion index value.
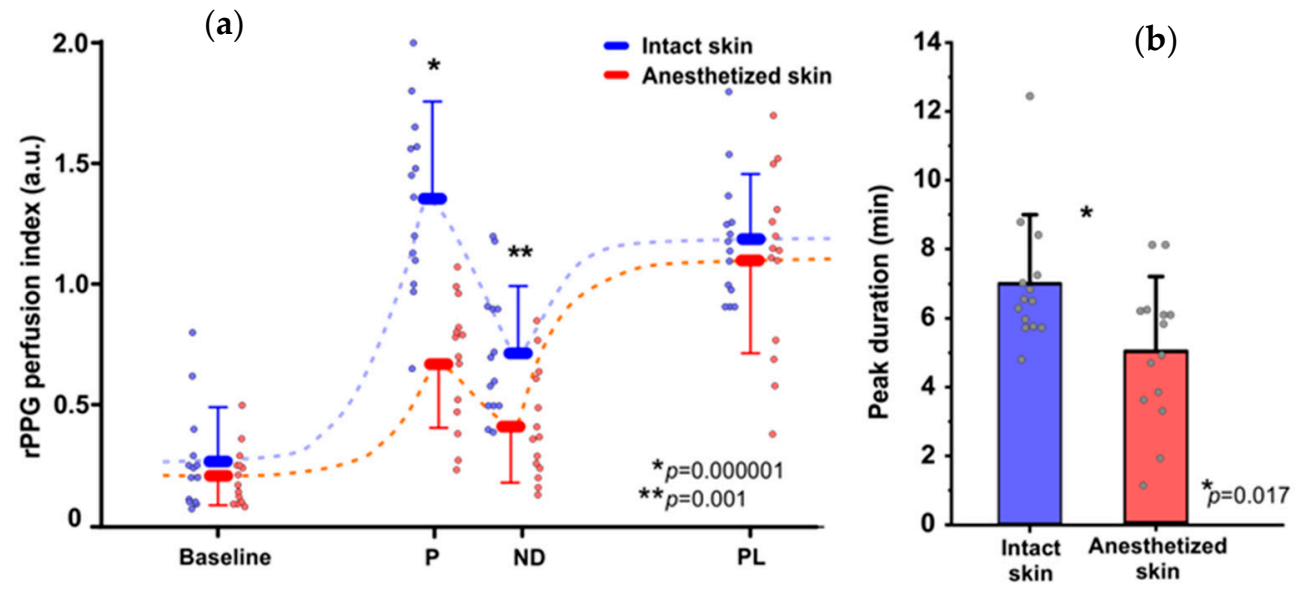

Figure 5. (a)-Topical skin heating induced vasomotor response in intact and anesthetized skin, group ( $\mathrm{n}=14)$ mean data \pm S.E.M., subjects individual measurements marked with small circles; (b)-Topical skin heating induced vasomotor response peak duration, group $(\mathrm{n}=14)$ mean data \pm S.E.M. from intact and EMLA anesthetized skin regions, subjects individual measurements marked with black small circles. Statistically significant difference denoted by asterisks. 


\subsection{Influence of EMLA on Cutaneous Flowmotions}

The average spectral power of flowmotions obtained from intact and EMLA treated skin region in three frequency ranges were presented in the bar chart in Figure 6. Unaffected skin exhibited relatively larger and statistically significant (One Way RM ANOVA; $p=0.001$ ) power for endothelial $(0.28 \pm 0.17$ a.u. $)$ and neurogenic $(0.25 \pm 0.13$ a.u. $)$ frequency ranges in comparison to myogenic ( $0.15 \pm 0.09$ a.u. $)$.

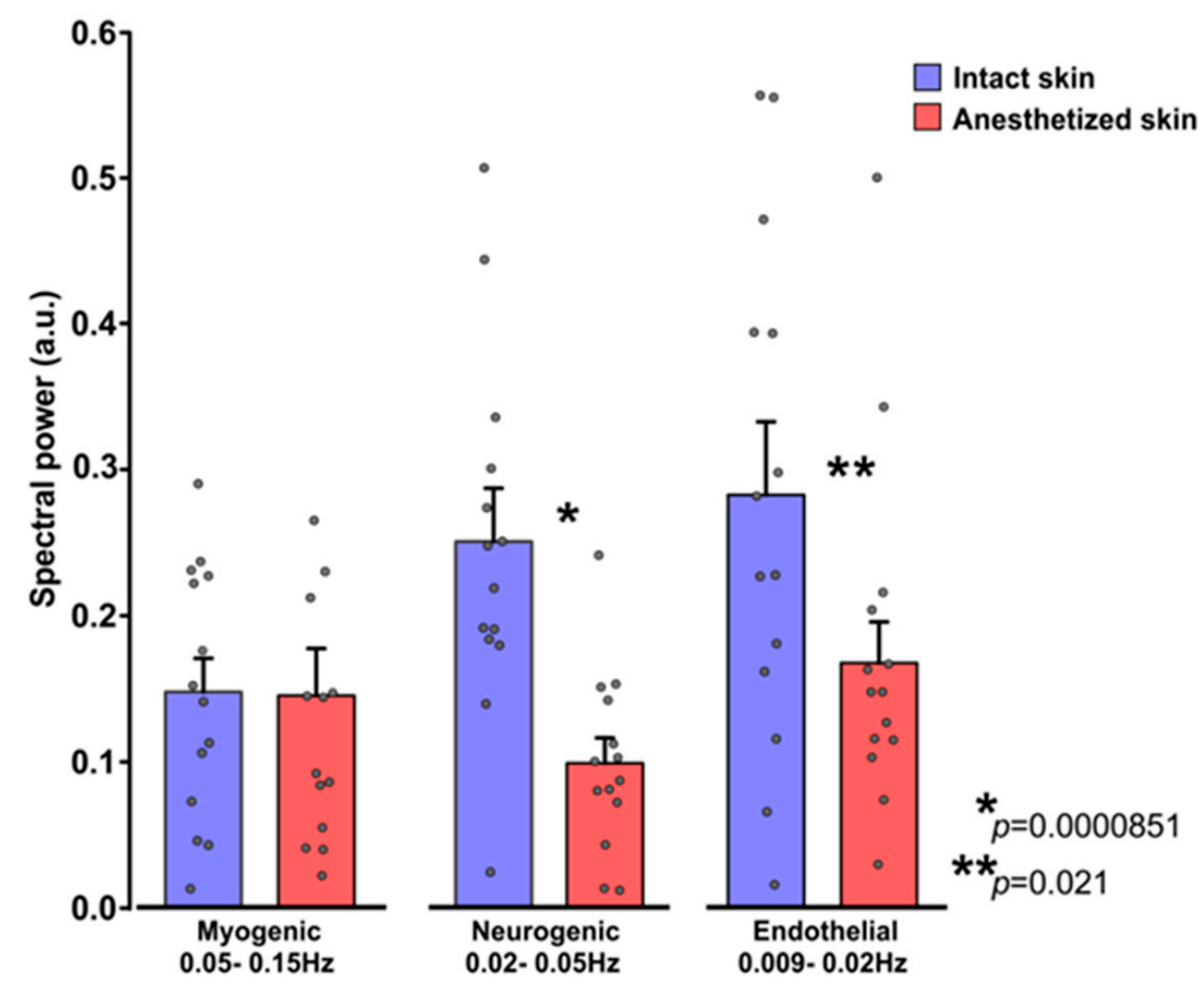

Figure 6. Cutaneous flowmotions obtained from intact and anesthetized skin regions, Group ( $\mathrm{n}=14)$ mean data \pm S.E.M., small black circles depict subjects' individual measurements; Statistically significant differences denoted by asterisks.

Different spectral power values were observed in EMLA treated skin-the spectral power of neurogenic $(0.10 \pm 0.06$ a.u. $)$ and endothelial $(0.17 \pm 0.10$ a.u.) frequency ranges were substantially lowered (neurogenic for $\sim 61 \%$, endothelial for $\sim 41 \%$ ) compared to intact skin region flowmotions. However, for the myogenic band ( $0.15 \pm 0.12$ a.u.), no difference was noted between intact and EMLA treated skin site.

Taken together, these results indicate that topical application EMLA gel to the skin significantly diminishes neurogenic and endothelial cutaneous flowmotions in remote photoplethysmography signal.

\section{Discussion}

The present study is a substantial extension of an earlier study presented at the conference materials [39] and its novelty is related to the development of protocol resembling derangement of small fiber function, similar to that observed in neuropathic patients. EMLA treated and intact skin regions were heated simultaneously with the same heating probe covering both regions, which allows us to capture different vasomotor responses using the same contactless photoplethysmography system, simultaneously, in very proximal cutaneous regions.

Whereas, in other studies using Laser Doppler techniques, either separate heater probes are placed on different limbs [40,41], or the measurement is performed from the 
same site but at different days- applying EMLA on one day and recording control the following day [42]. The detailed interpretation of our present findings is provided below.

\subsection{Vasomotor Responses}

The topical heating evoked vasomotor response obtained from intact skin region has a large similarity to that obtained in our earlier studies using contactless photoplethysmography technique [29], and laser Doppler registered response reported by other authors [43,44]. Laser Doppler studies suggest that during topical skin heating cutaneous hyperemia occurs [45], characterized by an initial peak within the first 5-6 min and a subsequent nadir followed by a sustained plateau [43]. Laser Doppler Imaging studies suggest that the peak is caused by an axon reflex, mediated by TRPV1 channel dependent activation of C-fiber afferent neurons that release substance $\mathrm{P}$ and calcitonin gene-related peptide (GCRP), with a modest contribution of $\mathrm{NO}$ [46] and may reflect both endothelial and small nerve fiber function $[47,48]$. That can explain the observation that EMLA treated skin exhibited a diminished initial peak, nadir and duration of initial peak, but not plato phase during the heat-induced vasomotor response. It is thought that the origin of plato phase is 60 to $70 \%$ NO-dependent, modulated by adenosine receptors, TRPV1 channels and reactive oxygen species [45]. A similar effect on initial peak duration was reported in other studies using Laser Doppler flowmetry, suggesting that topical application of lidocaine containing EMLA gel may change perfusion rise time [49,50]. The possibility of the direct influence of lidocaine as a major component of EMLA gel on cutaneous vasculature cannot be excluded, because of its vasodilator properties, possibly by the release of nitric oxide [51]. Nevertheless, this effect was not observed in our study using contactless photoplethysmography, as basal perfusion of EMLA anesthetized and intact skin regions did not substantially differ. The likely dilatory effect of lidocaine is masked by inhibition of cutaneous nerve fibers which dominate in regulation of cutaneous vascular tone. All subjects responded to EMLA gel and showed a similar trend of topical heating induced vasomotor response; however, the magnitude of response (time and amplitude of initial peak, nadir and plato phase) substantially differed across the subjects, possibly due to the inter-subject differences such as age, sex, and possibly vasoreactivity.

Another marker of cutaneous nerve fiber functions assessed in our study is the socalled vasomotor flare response, which manifests as the reddening of the skin outside the directly heated skin region. The evaluation of flare response as a parameter of vasomotor reactivity is widely used in different studies on cutaneous vasculature, using different provocation factors [48], such as Ach iontophoresis, electrical stimulation and topical skin heating; however, these studies mostly use Laser Doppler techniques (flowmetry or imaging) and cannot be directly attributed to photoplethysmographic data, as these techniques have different principles. The principle LDI is well defined, representing frequency change that light undergoes when reflected by red blood cells, thus output value is determined by flux-product of red blood cell linear velocity and concentration [52]. The same cannot be entirely said about PPG. The genesis of photoplethysmographic signal is not completely elucidated, hence modulation of PPG light intensity may be attributed to the mechanical movement of the capillary bed caused by varying arterial transmural pressure [53] and changes in RBCs orientation in the capillaries [54]. Nevertheless, studies suggest that PPG and LDI may share similar biophysics [55]. Our results confirm a decrease of flare area in EMLA treated skin, which is in line with other studies using Laser Doppler Imaging technique, suggesting a transitory suppressor effect of Lidocaine on the cutaneous sympathetic nerve fibers [56,57]. In the intact skin, topical heating depolarizes small unmyelinated dermal $C$ fibers, which results in afferent action potentials that are conducted towards the spinal cord and at branching points antidromically activate peripheral branches, adjacent to the initial stimulation point [58]. This triggers the release of vasoactive substances, such as substance $P$ and calcitonin gene-related peptide (CGRP), from nerve terminals and leads to a relaxation of arteriolar smooth muscle-vasodilation, resulting in increased perfusion at highly localized skin area, so-called flares [57]. While in 
anesthetized skin, these mechanisms are largely abolished leading to reduced flare area. Taken together these results confirm the role of neurogenic mechanisms in the manifestation of topical heating induced flare response, supporting other studies using laser Doppler flowmetry technique $[20,22,40,59,60]$.

One issue observed in our data is large inter-subject variance of vasomotor responses, possibly caused by superimposition of several independent factors, such as a level of emotional stress, body composition, and phase of the menstrual cycle in female subjects or other confounding factors. Another issue is regarding comparison of our findings to those reported in other studies, which is rather difficult because of the diversity of protocols and used instrumentation, as the majority of existing studies used Laser Doppler Flowmetry or Laser Doppler Imaging techniques, which are different from photoplethysmography.

\subsection{Cutaneous Flowmotions}

Alongside provocation tests, which through selective stimulus can trigger blood perfusion response attributed to the particular physiological mechanism, another approach is the assessment of circulatory function in the baseline using so-called flowmotions [61]. Flowmotions are rhythmic oscillations in blood flow, to date widely assessed by Laser Doppler flowmetry, hence there are few studies reporting the use of contact manner reflection photoplethysmography $[18,48,62]$. The current exact genesis of these oscillations is unknown, but existing studies suggest the local origin as the dynamic interaction of sympathetic vasoconstriction, pressure-dependent vasoconstriction, flow-dependent endothelium-mediated vasodilation, metabolic vasodilation, and spontaneous myogenic activity [63].

The marked finding of the present study is the decrease of neurogenic and endothelial component in the EMLA treated skin, which is supported by Doppler studies on Diabetic patients with neuropathy [17]. Another interesting observation indicated the resistance of the myogenic component to EMLA treatment. Several other studies on neuropathic patients confirmed similar effects $[17,63]$, thus we can speculate that EMLA treatment largely resembles dysfunction of cutaneous nerve fibers observed in neuropathic patients. However, to the best of our knowledge, there were no studies directly revealing the effect of EMLA gel on cutaneous flowmotions registered by photoplethysmography.

Summarizing, our results indicates that photoplethysmography derived cutaneous perfusion changes during vasomotor responses are similar to that reported by other studies using laser Doppler technique; however, the question remains whether the contactless modality of photoplethysmography could potentially substitute the gold standard techniques such as Laser Doppler imaging. Therefore, further studies should address this question.

\subsection{Limitations of the Study}

Despite the careful planning of the experimental design and the accurate implementation of all procedures, there are still several limitations that may potentially interfere with the present findings and therefore results should be interpreted with precaution.

The major limitation is related to the relatively small $n=14$ and heterogeneous study group (20-40 y). On one hand, the selection of a small and heterogeneous group was dictated by the present situation with global Coronavirus pandemics. Therefore, meaning a minimal number of highly motivated local University staff were enrolled to follow strict epidemiological requirements. On the other hand, it was interesting to see whether observed effects are manifested in a relatively heterogeneous sample comprising subjects of different sex and age. We believe that the observed inter subject variability is related mostly to the subjects individual differences, such as: age [64], unknown status of former smokers [65], daily use of coffee and beverages [66], level of emotional stress during measurement procedure [67], degree of physical fitness [68] and unknown status of the menstrual cycle in female subjects [69]. Nevertheless, while the size of this preliminary study was not very large, it was sufficient to highlight statistically significant results in a largely heterogeneous group. 
Another limitation is the lack of reference methods in the present study such as Laser Doppler, which is considered "the gold standard" for cutaneous vasomotor responses; therefore, it is difficult to compare the results with the other studies as they use Laser Doppler technique. There is ample evidence suggesting that cutaneous vasomotor responses registered simultaneously by both Laser Doppler technique and Photoplethysmography at $530 \mathrm{~nm}$ illumination have large similarities [28], which is also in line with other studies mentioning kinetics and pattern of topical heating induced vasomotor response obtained by laser Doppler $[41,57,70]$, which corresponds to that obtained by PPG [30].

The question which remains largely unanswered in the present study is regarding the feasibility of contactless photoplethysmography in the assessment of neuropathic patients. This has not been directly addressed, as healthy volunteers instead of neuropathic patients were enrolled as test subjects. Also, instead of natural pathophysiology of nerve fiber derangement during the disease course, temporal alterations of intact nerve fibers were evoked using topical cutaneous anesthesia, namely EMLA gel. Whether these alterations are attributable if not similar to that of neuropathic patients is not entirely clear, as the mechanisms of neuropathic pathophysiology are not fully elucidated. Nevertheless, topical anesthesia protocol has been used in several studies using Laser Doppler [40], and authors concluded that the application of local anesthesia produced temporary alterations of cutaneous somatosensory function, as well as vasomotor response with a similar manifestation observed in neuropathic patients. Another confirmation comes from our prior pilot study on neuropathic patients [29], highlighting on the decreased flare response, which supports the capability of photoplethysmography for examining neuropathic patients. Therefore, we believe that the accumulated evidence indirectly confirms the potential of contactless photoplethysmography for the assessment of nerve fiber function in neuropathic patients. However, more studies are required on neuropathic patients using contactless photoplethysmography.

\section{Conclusions}

The present results indicate the potential of the remote photoplethysmography in the assessment of cutaneous small nerve fiber function as supported by EMLA anesthesia protocol. Therefore, it is believed that along with the existing gold standard techniques such as laser Doppler Imaging, the contactless modality of photoplethysmography in the future could be used for examination of neuropathic patients as a cost-effective and affordable alternative. However, more extensive studies are required on this subject.

Supplementary Materials: The following are available online at https:/ / www.mdpi.com/1424-822 $0 / 21 / 4 / 1272 /$ s1.

Author Contributions: The work described in this article is the collaborative contribution of all authors. Conceptualization, writing-original draft preparation, Z.M.; methodology and formal analysis, A.A.; software, visualization and signal processing, U.R.; project administration, supervision, funding acquisition, manuscript editing, A.G. All authors have read and agreed to the published version of the manuscript.

Funding: This research was funded by Latvian Council of Science, grant number LZP-2018/1-0188.

Institutional Review Board Statement: The study was conducted according to the guidelines of the Declaration of Helsinki, and approved by the Ethics Committee of University of Latvia, Institute of Cardiology and Regenerative Medicine (Prot. code: 11/5, date of approval 3 May 2018).

Informed Consent Statement: Written informed consent was obtained from all subjects involved in the study.

Data Availability Statement: The data presented in this study are available in article Supplementary Files.

Acknowledgments: This study was supported by University of Latvia Institute of Atomic Physics and Spectroscopy, Biophotonics laboratory and Department of Human and animal Physiology, Faculty of Biology, University of Latvia. 
Conflicts of Interest: The authors declare no conflict of interest. The funders had no role in the design of the study; in the collection, analyses, or interpretation of data; in the writing of the manuscript, or in the decision to publish the results.

\section{References}

1. Al-Shekhlee, A.; Chelimsky, T.C.; Preston, D.C. Review: Small-fiber neuropathy. Neurologist 2002, 8, 237-253. [CrossRef] [PubMed]

2. Hovaguimian, A.; Gibbons, C.H. Diagnosis and treatment of pain in small-fiber neuropathy. Curr. Pain Headache Rep. 2011, 15, 193-200. [CrossRef]

3. Truini, A. A Review of Neuropathic Pain: From Diagnostic Tests to Mechanisms. Pain Ther. 2017, 6, 5-9. [CrossRef] [PubMed]

4. Callaghan, B.C.; Price, R.S.; Feldman, E.L. Distal Symmetric Polyneuropathy: A Review. JAMA 2015, 314, 2172-2181. [CrossRef] [PubMed]

5. Koralnik, I.J.; Tyler, K.L. COVID-19: A Global Threat to the Nervous System. Ann. Neurol. 2020, 88, 1-11. [CrossRef] [PubMed]

6. Lahiri, D.; Ardila, A. COVID-19 Pandemic: A Neurological Perspective. Cureus 2020, 12, e7889. [CrossRef] [PubMed]

7. Tsivgoulis, G.; Palaiodimou, L.; Katsanos, A.H.; Caso, V.; Köhrmann, M.; Molina, C.; Cordonnier, C.; Fischer, U.; Kelly, P.; Sharma, V.K.; et al. Neurological manifestations and implications of COVID-19 pandemic. Ther. Adv. Neurol. Disord. 2020, 13. [CrossRef]

8. Zaslansky, R.; Yarnitsky, D. Clinical applications of quantitative sensory testing (QST). J. Neurol. Sci. 1998, 153, 215-238. [CrossRef]

9. McCarthy, B.G.; Hsieh, S.T.; Stocks, A.; Hauer, P.; Macko, C.; Cornblath, D.R.; Griffin, J.W.; McArthur, J.C. Cutaneous innervation in sensory neuropathies: Evaluation by skin biopsy. Neurology 1995, 45, 1848-1855. [CrossRef]

10. Myers, M.I.; Peltier, A.C. Uses of skin biopsy for sensory and autonomic nerve assessment. Curr. Neurol. Neurosci. Rep. 2013, 13, 323. [CrossRef] [PubMed]

11. Roosterman, D.; Goerge, T.; Schneider, S.W.; Bunnett, N.W.; Steinhoff, M. Neuronal Control of Skin Function: The Skin as a Neuroimmunoendocrine Organ. Physiol. Rev. 2006, 86, 1309-1379. [CrossRef] [PubMed]

12. Slominski, A.; Wortsman, J.; Paus, R.; Elias, P.M.; Tobin, D.J.; Feingold, K.R. Skin as an endocrine organ: Implications for its function. Drug Discov. Today. Dis. Mech. 2008, 5, 137-144. [CrossRef]

13. Rigopoulos, D.; Larios, G.; Katsambas, A. Skin signs of systemic diseases. Clin. Dermatol. 2011, 29, 531-540. [CrossRef]

14. Bashkatov, A.N.; Genina, E.A.; Kochubey, V.I.; Tuchin, V. V Optical properties of human skin, subcutaneous and mucous tissues in the wavelength range from 400 to $2000 \mathrm{~nm}$. J. Phys. D Appl. Phys. 2005, 38, 2543-2555. [CrossRef]

15. Boer, M.; Duchnik, E.; Maleszka, R.; Marchlewicz, M. Structural and biophysical characteristics of human skin in maintaining proper epidermal barrier function. Postep. Dermatol. Alergol. 2016, 33, 1-5. [CrossRef]

16. Mignon, C.; Tobin, D.J.; Zeitouny, M.; Uzunbajakava, N.E. Shedding light on the variability of optical skin properties: Finding a path towards more accurate prediction of light propagation in human cutaneous compartments. Biomed. Opt. Express 2018, 9 , 852-872. [CrossRef]

17. Sun, P.C.; Kuo, C.D.; Chi, L.Y.; Lin, H.D.; Wei, S.H.; Chen, C.S. Microcirculatory vasomotor changes are associated with severity of peripheral neuropathy in patients with type 2 diabetes. Diabetes Vasc. Dis. Res. 2013, 10, 270-276. [CrossRef]

18. Mizeva, I.; Di Maria, C.; Frick, P.; Podtaev, S.; Allen, J. Quantifying the correlation between photoplethysmography and laser Doppler flowmetry microvascular low-frequency oscillations. J. Biomed. Opt. 2015, 20, 37007. [CrossRef]

19. Peltier, A.; Smith, A.G.; Russell, J.W.; Sheikh, K.; Bixby, B.; Howard, J.; Goldstein, J.; Song, Y.; Wang, L.; Feldman, E.L.; et al Reliability of quantitative sudomotor axon reflex testing and quantitative sensory testing in neuropathy of impaired glucose regulation. Muscle Nerve 2009, 39, 529-535. [CrossRef] [PubMed]

20. Ysihai, R. Abnormal LDIflare but Normal Quantitative Sensory Testing and Dermal Nerve Fiber Density in Patients with Painful Diabetic Neuropathy. Diabetes Care 2009, 32, 451-455. [CrossRef]

21. Sharma, S.; Venkitaraman, R.; Vas, P.R.J.; Rayman, G. Assessment of chemotherapy-induced peripheral neuropathy using the LDIFLARE technique: A novel technique to detect neural small fiber dysfunction. Brain Behav. 2015, 5, e00354. [CrossRef]

22. Namer, B.; Pfeffer, S.; Handwerker, H.O.; Schmelz, M.; Bickel, A. Axon reflex flare and quantitative sudomotor axon reflex contribute in the diagnosis of small fiber neuropathy. Muscle Nerve 2013, 47, 357-363. [CrossRef]

23. Kubasch, M.L.; Kubasch, A.S.; Torres Pacheco, J.; Buchmann, S.J.; Illigens, B.M.-W.; Barlinn, K.; Siepmann, T. Laser Doppler Assessment of Vasomotor Axon Reflex Responsiveness to Evaluate Neurovascular Function. Front. Neurol. 2017, 8, 370. [CrossRef]

24. Green, A.Q.; Krishnan, S.T.; Rayman, G. C-fiber function assessed by the laser doppler imager flare technique and acetylcholine iontophoresis. Muscle Nerve 2009, 40, 985-991. [CrossRef]

25. Hop, M.J.; Stekelenburg, C.M.; Hiddingh, J.; Kuipers, H.C.; Middelkoop, E.; Nieuwenhuis, M.K.; Polinder, S.; van Baar, M.E. Cost-Effectiveness of Laser Doppler Imaging in Burn Care in The Netherlands: A Randomized Controlled Trial. Plast. Reconstr. Surg. 2016, 137, 166e-176e. [CrossRef]

26. Sun, Y.; Papin, C.; Azorin-Peris, V.; Kalawsky, R.; Greenwald, S.; Hu, S. Use of ambient light in remote photoplethysmographic systems: Comparison between a high-performance camera and a low-cost webcam. J. Biomed. Opt. 2012, 17, 037005. [CrossRef]

27. Rubins, U.; Spigulis, J.; Miscuks, A. Application of Colour Magnification Technique for Revealing Skin Microcirculation Changes Under Regional Anaesthetic Input. In Proceedings of the 1st International Conference "Biophotonics Riga 2013", Riga, Latvia, 26-31 August 2013; Volume 9032, p. 903203.

28. Marcinkevics, Z.; Rubins, U.; Zaharans, J.; Miscuks, A.; Urtane, E.; Ozolina-Moll, L. Imaging photoplethysmography for clinical assessment of cutaneous microcirculation at two different depths. J. Biomed. Opt. 2016, 21, 35005. [CrossRef] 
29. Rubins, U.; Marcinkevics, Z.; Logina, I.; Grabovskis, A.; Kviesis-Kipge, E. Imaging photoplethysmography for assessment of chronic pain patients. In Optical Diagnostics and Sensing XIX: Toward Point-of-Care Diagnostics; Coté, G.L., Ed.; SPIE: Bellingham, WA, USA, 2019; Volume 10885, p. 8.

30. Marcinkevics, Z.; Rubins, U.; Aglinska, A.; Caica, A.; Grabovskis, A. Remote photoplethysmography for skin perfusion monitoring using narrowband illumination. In Clinical and Preclinical Optical Diagnostics II; Brown, J.Q., van Leeuwen, T.G., Eds.; SPIE: Bellingham, WA, USA, 2019; Volume 11073, p. 12.

31. Kamshilin, A.; Belaventseva, A.; Romashko, R.; Kulchin, Y.; Mamontov, O. Local Thermal Impact on Microcirculation Assessed by Imaging Photoplethysmography. Biol. Med. 2016, 8, 361. [CrossRef]

32. Volynsky, M.A.; Margaryants, N.B.; Mamontov, O.V.; Kamshilin, A.A. Contactless Monitoring of Microcirculation Reaction on Local Temperature Changes. Appl. Sci. 2019, 9, 4947. [CrossRef]

33. Rubins, U.; Miscuks, A.; Lange, M. Simple and convenient remote photoplethysmography system for monitoring regional anesthesia effectiveness. IFMBE Proc. 2016, 65, 378-381. [CrossRef]

34. World Medical Association World Medical Association Declaration of Helsinki. JAMA 2013, 310, 2191. [CrossRef]

35. Kastrup, J.; Bülow, J.; Lassen, N.A. Vasomotion in human skin before and after local heating recorded with laser Doppler flowmetry. A method for induction of vasomotion. Int. J. Microcirc. Clin. Exp. 1989, 8, 205-215.

36. Söderström, T.; Stefanovska, A.; Veber, M.; Svensson, H. Involvement of sympathetic nerve activity in skin blood flow oscillations in humans. Am. J. Physiol. Heart Circ. Physiol. 2003, 284, H1638-H1646. [CrossRef]

37. Kvernmo, H.D.; Stefanovska, A.; Kirkeboen, K.A.; Kvernebo, K. Oscillations in the human cutaneous blood perfusion signal modified by endothelium-dependent and endothelium-independent vasodilators. Microvasc. Res. 1999, 57, 298-309. [CrossRef]

38. Kvandal, P.; Stefanovska, A.; Veber, M.; Kvernmo, H.D.; Kirkebøen, K.A. Regulation of human cutaneous circulation evaluated by laser Doppler flowmetry, iontophoresis, and spectral analysis: Importance of nitric oxide and prostaglandines. Microvasc. Res. 2003, 65, 160-171. [CrossRef]

39. Marcinkevics, Z.; Aglinska, A.; Rubins, U.; Mikale, A.; Grabovskis, A. Imaging photoplethysmography for evaluation of cutaneous sensory nerve fiber function. In Biophotonics—Riga 2020; Spigulis, J., Ed.; SPIE: Bellingham, WA, USA, 2020; Volume 11585, pp. 44-49.

40. Krishnan, S.T.M.; Rayman, G. The LDIflare: A novel test of C-fiber function demonstrates early neuropathy in type 2 diabetes. Diabetes Care 2004, 27, 2930-2935. [CrossRef] [PubMed]

41. Metzler-Wilson, K.; Kellie, L.A.; Tomc, C.; Simpson, C.; Sammons, D.; Wilson, T.E. Differential vasodilatory responses to local heating in facial, glabrous and hairy skin. Clin. Physiol. Funct. Imaging 2012, 32, 361-366. [CrossRef] [PubMed]

42. Nieuwenhoff, M.D.; Wu, Y.; Huygen, F.J.P.M.; Schouten, A.C.; van der Helm, F.C.T.; Niehof, S.P. Reproducibility of axon reflex-related vasodilation assessed by dynamic thermal imaging in healthy subjects. Microvasc. Res. 2016, 106, 1-7. [CrossRef] [PubMed]

43. Ciplak, M.; Pasche, A.; Heim, A.; Haeberli, C.; Waeber, B.; Liaudet, L.; Feihl, F.; Engelberger, R. The vasodilatory response of skin microcirculation to local heating is subject to desensitization. Microcirculation 2009, 16, 265-275. [CrossRef] [PubMed]

44. Martin, Z.T.; Shannon, C.A.; Kistler, B.M.; Nagelkirk, P.R.; Del Pozzi, A.T. Effect of Sex and Menstrual Cycle on Skin Sensory Nerve Contribution to Local Heating. Int. J. Exerc. Sci. 2019, 12, 1265-1279. [PubMed]

45. Huang, C.; Wang, S.; Tsai, Y. Axon reflex-related hyperemia induced by short local heating is reproducible. Microvasc. Res. 2012, 84, 351-355. [CrossRef]

46. Marche, P.; Dubois, S.; Abraham, P.; Gascoin, L. Neurovascular microcirculatory vasodilation mediated by C-fibers and Transient receptor potential vanilloid-type-1 channels (TRPV 1) is impaired in type 1 diabetes. Nat. Publ. Gr. 2017, 1-9. [CrossRef] [PubMed]

47. Wong, B.J.; Hollowed, C.G. Current concepts of active vasodilation in human skin. Temperature 2017, 4, 41-59. [CrossRef] [PubMed]

48. Hijazi, M.M.; Buchmann, S.J.; Sedghi, A.; Illigens, B.M.; Reichmann, H.; Schackert, G.; Siepmann, T. Assessment of cutaneous axon-reflex responses to evaluate functional integrity of autonomic small nerve fibers. Neurol. Sci. Off. J. Ital. Neurol. Soc. Ital. Soc. Clin. Neurophysiol. 2020, 41, 1685-1696. [CrossRef]

49. Arildsson, M.; Nilsson, G.E.; Strömberg, T. Effects on skin blood flow by provocation during local analgesia. Microvasc. Res. 2000, 59, 122-130. [CrossRef] [PubMed]

50. Caselli, A.; Uccioli, L.; Khaodhiar, L.; Veves, A. Local anesthesia reduces the maximal skin vasodilation during iontophoresis of sodium nitroprusside and heating. Microvasc. Res. 2003, 66, 134-139. [CrossRef]

51. Newton, D.J.; McLeod, G.A.; Khan, F.; Belch, J.J.F. Mechanisms influencing the vasoactive effects of lidocaine in human skin. Anaesthesia 2007, 62, 146-150. [CrossRef] [PubMed]

52. Murray, A.K.; Herrick, A.L.; King, T.A. Laser Doppler imaging: A developing technique for application in the rheumatic diseases Rheumatology 2004, 43, 1210-1218. [CrossRef]

53. Kamshilin, A.A.; Nippolainen, E.; Sidorov, I.S.; Vasilev, P.V.; Erofeev, N.P.; Podolian, N.P.; Romashko, R.V. A new look at the essence of the imaging photoplethysmography. Sci. Rep. 2015, 5, 10494. [CrossRef]

54. Volkov, M.V.; Margaryants, N.B.; Potemkin, A.V.; Volynsky, M.A.; Gurov, I.P.; Mamontov, O.V.; Kamshilin, A.A. Video capillaroscopy clarifies mechanism of the photoplethysmographic waveform appearance. Sci. Rep. 2017, 7, 13298. [CrossRef] 
55. Silva, H.; Bento, M.; Vieira, H.; Monteiro Rodrigues, L. Comparing the spectral components of laser Doppler flowmetry and photoplethysmography signals for the assessment of the vascular response to hyperoxia. J. Biomed. Biopharm. Res. 2017, 14, 187-194. [CrossRef]

56. Minson, C.T.; Berry, L.T.; Joyner, M.J. Nitric oxide and neurally mediated regulation of skin blood flow during local heating. J. Appl. Physiol. 2001, 91, 1619-1626. [CrossRef]

57. Carter, S.J.; Hodges, G.J. Sensory and sympathetic nerve contributions to the cutaneous vasodilator response from a noxious heat stimulus. Exp. Physiol. 2011, 96, 1208-1217. [CrossRef]

58. Weidner, C.; Schmidt, R.; Schmelz, M.; Torebjork, H.E.; Handwerker, H.O. Action potential conduction in the terminal arborisation of nociceptive C-fibre afferents. J. Physiol. 2003, 547, 931-940. [CrossRef]

59. Bickel, A.; Heyer, G.; Senger, C.; Maihöfner, C.; Maihoefner, C.; Heuss, D.; Hilz, M.J.; Namer, B. C-fiber axon reflex flare size correlates with epidermal nerve fiber density in human skin biopsies. J. Peripher. Nerv. Syst. 2009, 14, 294-299. [CrossRef]

60. Illigens, B.M.W.; Siepmann, T.; Roofeh, J.; Gibbons, C.H. Laser Doppler imaging in the detection of peripheral neuropathy. Auton. Neurosci. Basic Clin. 2013, 177, 286-290. [CrossRef]

61. Aalkjær, C.; Boedtkjer, D.; Matchkov, V. Vasomotion-What is currently thought? Acta Physiol. 2011, 202, 253-269. [CrossRef]

62. Rodrigues, L.M.; Rocha, C.; Ferreira, H.; Silva, H. Different lasers reveal different skin microcirculatory flowmotion-data from the wavelet transform analysis of human hindlimb perfusion. Sci. Rep. 2019, 9, 16951. [CrossRef]

63. Rossi, M.; Carpi, A.; Galetta, F.; Franzoni, F.; Santoro, G. The investigation of skin blood flowmotion: A new approach to study the microcirculatory impairment in vascular diseases? Biomed. Pharmacother. 2006, 60, 437-442. [CrossRef]

64. Hodges, G.J.; Mallette, M.M.; Tew, G.A.; Saxton, J.M.; Moss, J.; Ruddock, A.D.; Klonizakis, M. Effect of age on cutaneous vasomotor responses during local skin heating. Microvasc. Res. 2017, 112, 47-52. [CrossRef]

65. Edvinsson, M.-L.; Andersson, S.E.; Xu, C.-B.; Edvinsson, L. Cigarette smoking leads to reduced relaxant responses of the cutaneous microcirculation. Vasc. Health Risk Manag. 2008, 4, 699-704. [CrossRef]

66. Tesselaar, E.; Nezirevic Dernroth, D.; Farnebo, S. Acute effects of coffee on skin blood flow and microvascular function. Microvasc. Res. 2017, 114, 58-64. [CrossRef] [PubMed]

67. Glazachev, O.S.; Dudnik, E.N. The microcirculatory blood flow and autonomic regulation's functional state in young people with different levels of subjectively experienced psychological stress. Fiziol. Cheloveka 2012, 38, 50-57.

68. Simmons, G.H.; Wong, B.J.; Holowatz, L.A.; Kenney, W.L. Changes in the control of skin blood flow with exercise training: Where do cutaneous vascular adaptations fit in? Exp. Physiol. 2011, 96, 822-828. [CrossRef]

69. Petrofsky, J.; Lee, H.; Khowailed, I.A. Sudomotor and vasomotor activity during the menstrual cycle with global heating. Clin. Physiol. Funct. Imaging 2017, 37, 366-371. [CrossRef] [PubMed]

70. Minson, C.T.; Tew, G.A.; Klonizakis, M.; Moss, J.; Ruddock, A.D.; Saxton, J.M.; Gary, J. Thermal provocation to evaluate microvascular reactivity in human skin Mechanisms and Modulators of Temperature Regulation Thermal provocation to evaluate microvascular reactivity in human skin. J. Appl. Physiol. 2011, 109, 1239-1246. [CrossRef] [PubMed] 